\title{
Adaptive Transfer Functions in Radial Basis Function (RBF) Networks
}

\author{
Günther A. Hoffmann \\ Humboldt University Berlin, Department of Computer Science, \\ Rechnerorganisation und Kommunikation ROK, 10099 Berlin, Germany \\ gunho@informatik.hu-berlin.de
}

\begin{abstract}
The quality of Radial Basis Functions (RBF) and other nonlinear learning networks such as Multi Layer Perceptrons (MLP) depend significantly on issues in architecture, learning algorithms, initialisation heuristics and regularization techniques. Little attention has been given to the effect of mixture transfer functions in RBF networks on model quality and efficiency of parameter optimisation. We propose Universal Basis Functions (UBF) with flexible activation functions which are parameterised to change their shape smoothly from one functional form to another. This way they can cover bounded and unbounded subspaces depending on data distribution. We define UBF and apply them to a number of classification and function approximation tasks. We find that the UBF approach outperforms traditional RBF with the Hermite data set, a noisy Fourier series and a non $\varphi$-separable classification problem, however it does not improve statistically significant on the MackeyGlass chaotic time series. The paper concludes with comments and issues for future research.
\end{abstract}

\section{Introduction and Objectives}

The quality of Radial Basis Functions (RBF) and other nonlinear learning networks such as Multi Layer Perceptrons (MLP) depends significantly on architecture, learning algorithms, initialisation heuristics and regularization techniques. Little attention has been given to the effect of flexible transfer functions in RBF networks on model quality and efficiency of parameter optimisation. Even though Gaussian and sigmoidal transfer functions prevail in literature, there is little a priori reason why models solely based on these transfer function will provide optimal model quality. In this paper we investigate the effects of adaptive transfer functions, which replace the Gaussian transfer functions in RBF.

The rest of the paper is organized as follows: Before we describe the universal basis function (UBF) approach in more detail in chapter three we review previous attempts and the state of the art of universal transfer functions in chapter two. RBF have been described and reviewed extensively in literature [1][6][13]. We will not revisit this topic. We will briefly comment on the problem of parameter optimisation in chapter four. We will then investigate the effect of different types of transfer func- 
tions on selected problems such as the Hermite data set, the chaotic Mackey-Glass time series, a noisy Fourier series and a classification problem in chapter five. In chapter six we discuss our results and outlay future work.

\section{Related Work}

A straight forward integration of MLP and RBF was carried out in [3]. In [4] and [5] an extensive overview of transfer functions is given. Little practical expertise has been published. To the best of our knowledge a systematic comparison of transfer functions is yet missing.

\section{Universal Basis Functions: RBF with Mixed Transfer Functions}

We propose flexible kernels as in (2) to replace the standard Gaussian kernels (1) in RBF. We call this approach universal basis functions (UBF). These novel kernels can be parameterised to change their shape smoothly from one functional form to another. The Gaussian transfer function is a special case of UBF.

$$
G(r)=e^{-r^{2} / 2 \sigma^{2}}
$$

The kernels we propose consist of a mixture of two activation functions. To combine different types of activation functions in one kernel, additive and multiplicative mixtures have been proposed in [3][5]. We propose a mixture of activation functions by smoothly morphing one activation function into the other as in (2). We combine Gaussian (4), sigmoid (5) and multiquadratic (6) activation functions.

$$
G\left(\mathbf{x} ; \mathbf{r}, \boldsymbol{\sigma}, \omega^{\prime}\right)=\omega^{\prime} \Phi_{1}(\mathbf{x} ; \mathbf{r}, \boldsymbol{\sigma})+\left(1-\omega^{\prime}\right) \Phi_{2}(\mathbf{x} ; \mathbf{r}, \boldsymbol{\sigma})
$$

with

$$
\omega^{\prime}=\tanh \left(\frac{1}{2} \omega\right) ; \omega \in(-\infty, \infty)
$$

and $\Phi \in \Phi_{1 . .3}^{\prime}$

$$
\begin{aligned}
& \Phi_{1}^{\prime}=e^{-r^{2} / 2 \sigma^{2}} \\
& \Phi_{2}^{\prime}=\tanh \left(r / \sigma^{2}\right) \\
& \Phi_{3}^{\prime}=\sqrt{r^{2}+\sigma^{2}}
\end{aligned}
$$


If we do have information about the data distribution we can incorporate this knowledge by giving a bias towards a specific activation function by initialising the slider value $\omega^{\prime}$ accordingly. However, as frequently is the case knowledge about the data distribution is either difficult to obtain or not available at all. In this case we start with an educated guess. If no further information is available to justify a bias towards a particular activation function we set $\omega^{\prime}=0.5$.

\section{Parameter Optimisation}

In $\mathrm{UBF}$ we have to optimise the joint set of parameters $\Theta=\left\{t, \sigma, \omega^{\prime}\right\}$ with kernel positions $t$, kernel widths $\sigma$ and the UBF slider value $\omega^{\prime}$ which controls the shape of the kernels. We employ a global stochastic optimisation procedure, the derandomised Evolution Strategy with full covariance matrix adaptation (CMA-ES). This procedure is a stochastic optimisation scheme which has been described in detail in [7] and [14].

\section{Results}

Figure 1 depicts the mean square error of the classification problem as a function of the UBF slider value $\omega^{\prime}$. For a Gaussian RBF kernel network we get a straight line because the kernel shape does not change with a varying $\omega^{\prime}$. For a mixture UBF kernel approach we clearly get an optimal kernel shape minimizing the models classification error at $\omega^{\prime} \approx 0.2$ implying a mildly Gaussian bias.

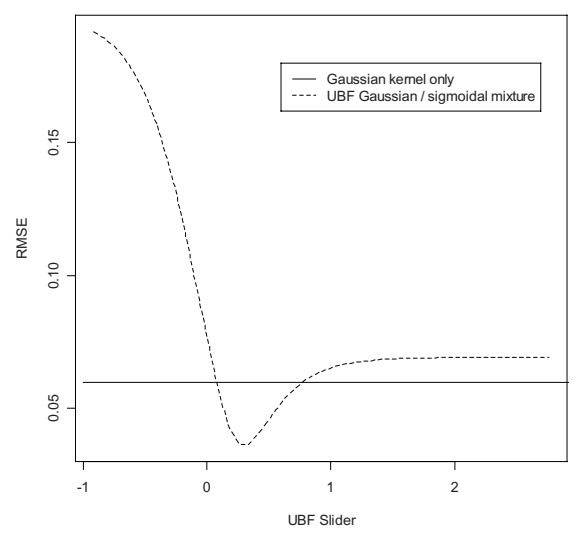

Fig. 1. The categorization error in terms of root-mean-square-errors (RMSE) is shown as the straight line for the RBF model. The UBF mixture model shows different errors as we smoothly change the activation function from Gaussian to sigmoidal. A UBF slider value larger than one, signals a strong Gaussian influence, a value below zero signals sigmoid influence. 


\section{Discussion and Future Work}

In this paper we have proposed Universal Basis Functions (UBF) as an extension to Radial Basis Functions (RBF). We investigated the models generalisation capabilities in terms of means square errors. We compared UBF and RBF. We find that classification of a non trivial dichotomy and function approximation of the Hermite data set and a noisy Fourier series the UBF approach significantly outperforms the RBF approach. We also find that for the Mackey-Glass data series the UBF approach does not significantly outperform the traditional RBF approach. UBF offer the possibility to adapt the transfer function locally to the search space. RBF are a special case of UBF. This is achieved by evolving a transfer function which is best suited for covering the local parameter space. The activation function $\Phi$ can thereby take on any form ranging smoothly from a $\Phi_{1}$ to a $\Phi_{2}$. For a Gaussian $\Phi_{1}=\Phi_{2}$ we get the traditional RBF network. If the data distribution is known the optimal activation function might be chosen based on analytical and theoretical consideration. However, in high dimensional space such knowledge about the data distribution might be difficult to obtain or not available at all. In these cases UBF might be a powerful extension of RBF. There are a number of open issues which we will address in future research. Further work is in progress to analyse the theoretical limits of UBF. Furthermore we will investigate how to reduce the complexity of the parameter space. Also currently we work with only one mixture value $\omega^{\prime}$ for all kernels and all dimensions. We expect a higher impact on model quality if we introduce a full $\omega^{\prime}$-matrix allowing for adaptation of the activation function for each kernel.

\section{References}

1. Bishop C. M. (1995) Neural Networks for Pattern Recognition; Clarendon Press

2. Cybenko G. (1989) Approximation by Superposition of a Sigmoidal Function; Mathematics of Control Signals and Systems 2, pp. 303-314

3. Dorffner G. (1994) A Unified Framework for MLPs and RBFNs: Introducing Conic Section Function Networks; Dept. of Medical Cybernetics and Artificial Intelligence University of Vienna

4. Duch W., Jankowski N. (1999) Survey of Neural Transfer Functions; Neural Computing Surveys 2,163-212

5. Duch W., Jankowski N. (2001) Transfer functions: hidden possibilities for better neural networks; ESANN'2001 proceedings - European Symposium on Artificial Neural Networks Bruges (Belgium), pp. 81-94

6. Girosi F., Jones M., Poggio T. (1993) Regularization Theory and Neural Networks; MIT Cambridge; AI Memo 1430

7. Hansen, Müller, Koumoutsakos (2003); Reducing the time complexity of the derandomized Evolution Strategy with Covariance Matrix Adaptation (CMA-ES); Evolutionary Computation 11(1):1-18, MIT Press

8. Hoffmann G. A. (1997b); Evolving Parameter Sets for Conditional Density Models; Intl. Symposium on Multi-Techn. Inf. Proc.; Taiwan 
9. Hutchinson James M., Lo Andrew, Poggio Tomaso (1994) A Nonparametric Approach to Pricing and Hedging Derivative Securities Via Learning Networks; MIT AI Memo No. 1471 or C.B.C.L. Paper No. 92

10. Jordan M. I., Bishop C. M. (1996); Neural Networks; AI Memo No. 1562; MIT

11. Lapedes A., Farber R. (1987) Nonlinear Signal Processing using Neural Networks; Prediction and System Modeling; Proceedings of Real Time Systems Symposium

12. Park J., Sandberg W. (1991); Universal Approximation using radial basis function networks; Neural Computation vol 3:(2):246-257

13. Poggio T., Girosi F. (1989); A Theory of Networks for Approximation and Learning; AI Memo 1140, MIT Cambridge

14. Ostermeier A., Gawelczyk A., Hansen N. (1993); A Derandomized Approach to Self Adaptation of Evolution Strategies; Technische Universität Berlin, Technical Report: TR-93003 